\title{
Obtención de carbón activado del endocarpio de shapaja (Attalea phalerata) procedente de la región San Martín, empleando un método físico
}

\author{
Obtaining activated carbon of shapaja endocarp (Attalea phalerata) proceeding \\ from San Martín region, using a physical method
}

\author{
María del Carmen Coronado Martínez ${ }^{1}$, Graciela Egoavil Cueva-Gálvez², \\ Héctor E. Gonzáles Mora ${ }^{3}$ y Pedro Carrasco Pérez ${ }^{4}$
}

\begin{abstract}
Resumen
En la presente investigación se evaluó el carbón activado por medios físicos a partir de semillas de "shapaja" (Attalea phalerata). Se analizaron los carbones obtenidos y se determinó la influencia de las variables: granulometría $(-10 /+20$ y -30/+40), temperatura $\left(800,900\right.$ y $\left.1000^{\circ} \mathrm{C}\right)$ y tiempo de activación (10, 20 y 30 minutos), sobre los carbones ensayados. De acuerdo a la evaluación realizada, se encontró que los carbones de la granulometría $-10 /+20$ (granular) y $-30 /+40$ (polvo), activados ambos a $900^{\circ} \mathrm{C}$ y 30 minutos, presentan un gran potencial para ser usados tanto en la industria azucarera como en tratamiento de agua. Los carbones de granulometría más fina $(-30 /+40)$, alcanzaron los valores más altos de porcentaje de absorción de azul de metileno, con valores promedio de 90,4 y 99,4 por ciento para $-10 /+20$ y $-30 /+40$, respectivamente. De acuerdo a los valores obtenidos, se evaluó la calidad de los carbones activados según Norma Técnica Peruana (NTP), para uso de carbón activado granular en el tratamiento de agua y la Norma Mexicana NMX-295-1981 para uso de carbón activado en la refinación de azúcar, con muy buenos resultados.
\end{abstract}

Palabras clave: shapaja; Attalea phalerata; carbón activado; activación; caracterización química.

\begin{abstract}
In this study we evaluated the activated carbon by physical means from "shapaja" seeds (Attalea phalerata). Carbons obtained were analyzed and determined the influence of variables: fineness $(-10 /+20-30 /+40)$, temperature $\left(800,900\right.$ and $\left.1000^{\circ} \mathrm{C}\right)$ and activation time (10, 20 and 30 minutes), the carbons tested. According to the evaluation, it was found that the particle size carbons $-10 /+20$ (granular) and $-30 /+40$ (dust), activated
\end{abstract}

\footnotetext{
${ }^{1}$ Consultor independiente, Perú.

${ }^{2}$ Facultad de Ciencias Forestales, Universidad Nacional Agraria La Molina, Perú. E-mail: gegoavil@lamolina.edu.pe

${ }^{3}$ Facultad de Ciencias Forestales, Universidad Nacional Agraria La Molina, Perú

${ }^{4}$ Peruvian Agribusiness Impact Fund, Perú.
} 
both at $900{ }^{\circ} \mathrm{C}$ and 30 minutes, has great potential to be used both in the sugar industry and in water treatment. The finer-grained carbon $(-30 /+40)$, obtained the highest percentage of methylene blue absorption, with average values of 90,4 and 99,4 percent for $-10 /+20$ and $-30 /+40$, respectively. According to the values obtained, we assessed the quality of the activated carbons as International Standard (NTP) for granular activated carbon used in water treatment and NMX-295-1981 activated carbon for use in the sugar refining, with very good results.

Key words: shapaja; Attalea phalerata; activated carbon; activation; chemical characterization.

\section{Introducción}

En el Perú, aproximadamente el 57\% del territorio nacional son áreas boscosas, con gran diversidad biológica expresado en los variados tipos de bosque y sus productos. En ellos se encuentra, una gran cantidad de productos no maderables (PFNM) que van creciendo en interés económico. El 2010 el Perú alcanzó un volumen de exportación de 163 millones de US\$ FOB, con tendencia creciente en el tiempo (MINAM 2011). Al respecto, en la Amazonía destacan las palmas o palmeras que por sus múltiples usos, constituyen el recurso vegetal con mayor utilidad entre los pobladores de la selva peruana, y cuyos usos se dan en la alimentación, material de construcción, artesanía, medicina, herramientas y utensilios. Sin embargo, muchas de ellas aun siendo especies multiuso, dejan residuos de uso potencial para otras industrias.

La "shapaja" (Attalea phalerata) es una especie muy utilizada en la Amazonía, de cuya almendra se puede obtener aceite para uso industrial, muchas veces generando ingresos a familias de escasos recursos. Reynel et al. (2003), describe a la shapaja (Attalea phalerata), como una palmera que se distribuye en el trópico desde centro y sur del Brasil hasta la amazonia de Perú y Bolivia, de preferencia en zonas con intervención antropogénica hacia altitudes de $1000 \mathrm{msnm}$. Por otro lado, los residuos de los frutos de shapaja son utilizados en la fabricación de carbón vegetal y su pulpa puede orientarse a la fabricación de etanol (Castro 2008). En el Perú aún no se registra el uso industrial de esta especie, pero sí de manera artesanal.
Fombuena (2009) señala que el carbón activado, es un producto extremadamente poroso pudiendo llegar a desarrollar áreas superficiales excepcionalmente altas, del orden de $1500 \mathrm{~m}^{2} / \mathrm{g}$ de carbón, facilitando la adsorción física de gases y vapores de mezclas gaseosas o sustancias dispersas en líquidos. Asimismo, Rodríguez (2004), señala que el tamaño y distribución de los poros en la estructura carbonosa pueden ser controlados para satisfacer necesidades de la tecnología actual y futura, mediante una presentación adecuada, esto es, granular y en polvo.

Respecto a su composición, Fombuena (2009), menciona que el carbón activado está formado por aproximadamente 75-80 \% de carbono, 5-10\% de cenizas, $60 \%$ de oxígeno y $0,5 \%$ de hidrógeno. Su estructura la conforman carbones muy desordenados e isótropos (Menéndez 2008), como un conjunto irregular de capas de carbono que presentan una ordenación al azar y entrecruzada, los espacios entre ellas constituyen la porosidad (Rodríguez 2004). Por la capacidad de adsorción los carbones activados, son utilizados en purificación de líquidos y gases (Menéndez 2008), de los cuales el $80 \%$ se emplean en el tratamiento de aguas (Rodríguez 2004), siendo los carbones en polvo los más indicados; sin embargo, los granulares pueden ser empleados en ambos casos, dependiendo del tipo de porosidad (Márquez et al. 2007).

En el Perú, el carbón activado se emplea en la recuperación de oro, en el proceso de "cianuración" (Sociedad Nacional de Minería, Petróleo y Energía 2006). Además, las estadísticas medioambientales del INEI (2011), reportan des- 
de el 2006, el empleo de carbón activado para tratamiento del agua potable en Lima Metropolitana.

La evaluación de las propiedades del carbón activado se realiza a través de un análisis proximal, del contenido de humedad, contenido de material volátil, cenizas y carbono fijo. Por otro lado la densidad aparente en promedio, de un carbón activado granular oscila entre 0,24 y $0,3 \mathrm{~g} / \mathrm{cm}^{3}$, para carbones comerciales, entre 0,3 a $0,7 \mathrm{~g} / \mathrm{cm}^{3}$ (U.S. Army Corps of Engineers 2001). Los valores de $\mathrm{pH}$ varían dependiendo del método de activación, los activados químicamente pueden presentar valores de $\mathrm{pH}$ muy ácidos por lo que se someten a un lavado, que puede neutralizar el carbón o incrementar los valores de $\mathrm{pH}$ (Performance Chemicals Inc. 2002). Para determinar si un carbón es de calidad, los resultados de su composición deben compararse con normas técnicas existentes, nacionales o internacionales, y de acuerdo al uso potencial que se quiera dar al carbón. Entre las especificaciones se encuentran la Norma Técnica Mexicana NMX-F-295-1981 (SCFI 1981), la Norma Técnica Peruana NTP 207.024:1982 (ITINTEC 1982) y las características del carbón comercial Calgon GRC 20 de la empresa Calgon Carbón Corporation.

Por otro lado, la producción del carbón activado es considerada una industria emergente por los recientes usos demostrados en métodos de recuperación de metales, tratamientos de aguas, adsorción de impurezas, entre otros, siendo estos dos últimos los de aplicación más destacada.

A nivel mundial, en el año 2004, se estimó un crecimiento significativo de hasta 1,7 millones, siendo Asia Pacífico la región con mayor demanda de carbón activado (Fredonia group 2010); debido principalmente a las iniciativas de protección ambiental. En el Perú también la demanda por el carbón activado va en aumento dada su utilidad en la industria minera y potabilización del agua. En el 2008 y 2009 se utilizaron aproximadamente $75 \mathrm{t}$ de carbón para el tratamiento de aguas en Lima Metropolitana (INEI 2011). Asimismo, en el año 2010, el Perú importó un valor mayor a 4 millones de dólares americanos (Trademap 2010).

Por tanto, los PFNM, en particular las palmeras y sus derivados, no son aprovechados en todo su potencial, limitados por la falta de investigaciones y desarrollo de mercados que permitan un manejo sostenible de los recursos y aporten a la rentabilidad de la industria forestal. La presente investigación busca evaluar la calidad del carbón activado de shapaja, mediante el método físico, sus propiedades físico químicas, la influencia del tipo de grano, temperatura y tiempo de activación.

\section{Materiales y Métodos}

El desarrollo experimental se realizó en el Laboratorio de Transformación Química del Departamento de Industrias Forestales de la Universidad Nacional Agraria La Molina (Lima, Perú), en el marco del convenio UNALM con la empresa Amazon Ivory E.I.R.L dentro del proyecto FINCyT - PITEI N 114.

La materia prima corresponde al endocarpio del fruto de shapaja procedente del distrito de Barranquita, provincia de Lamas, departamento de San Martín.

\section{Obtención del carbón activado}

Se dejó secar aproximadamente $10 \mathrm{~kg}$ de semillas de shapaja al medio ambiente hasta humedad de equilibrio. Se obtuvo su masa húmeda, contenido de humedad y masa seca.

Se realizó la carbonización a $500^{\circ} \mathrm{C}$, por 1,5 horas. Se dejó enfriar durante 24 horas, antes de su descarga. Se obtuvo la masa y el rendimiento del carbón vegetal.

Se procedió a la molienda y clasificación, mediante un molino de granos, mortero y tamices (ASTM, 10, 20, 30 y 40). Se seleccionaron dos fracciones: granular, entre - $10 /+20$, en un rango de 2 y $0,85 \mathrm{~mm}$; y polvo, entre $-30 /+40$, en un rango de 0,6 y $0,425 \mathrm{~mm}$. Denominado grano I y grano II respectivamente.

Para la activación, se preparó muestras de $7 \mathrm{~g}$ para ambas fracciones, se colocaron en crisoles de porcelana con tapa, y llevadas a un horno 
de activación de lecho fijo a 800, 900 y 1000 ${ }^{\circ} \mathrm{C}$, por 10,20 y 30 minutos, como agente de activación el aire. Se descargaron y enfriaron a temperatura ambiente por 30 minutos. Se determinó la masa seca y el rendimiento de activación.

\section{Evaluación del carbón vegetal y carbón ac- tivado}

Se empleó la norma ASTM D-1762 para la evaluación del contenido de humedad $(\mathrm{CH} \%)$, material volátil (MV\%), cenizas (CZ\%) y contenido de carbono fijo (CF\%), para las muestras de carbón vegetal y carbón activado

El rendimiento de carbón vegetal (Rcv \%) se determinó mediante la siguiente fórmula:

$R c v(\%)=$ masa del carbón vegetal $(g){ }^{*} 100 /$ masa seca del endocarpio de shapaja $(g)$

El Rendimiento de carbón activado (Rca \%) se determinó según la siguiente fórmula:

Rca $(\%)=$ masa del carbón activado $(g){ }^{\star} 100$ / masa seca del carbón vegetal $(g)$

La porosidad fue determinada según la fórmula propuesta por Dubini, modificada por Azañero (1988):

$$
G P=(100-R c a)
$$

Donde: $\mathrm{GP}=$ grado de porosidad

La densidad aparente $\left(\mathrm{g} / \mathrm{cm}^{3}\right)$ fue determinada según NTP-15-000-1982. El potencial de hidrógeno $(\mathrm{pH})$, según ASTM D 6851-02. Y la prueba de adsorción de azul de metileno, según Layseca (2001).

\section{Selección del carbón activado}

Se seleccionó cada tipo de grano que cumplió con lo señalado en las características descritas por NTP (1982), la norma técnica mexicana
NMX-F-295-1981 (SFCI 1981) y un carbón activado comercial a base de cáscara de coco (Calgon, GRC 20 - 6X12).

\section{Diseño experimental}

Según un arreglo factorial de $2 \times 3 \times 3$ bajo un diseño completamente al azar de un solo bloque con tres repeticiones. Las variables independientes son: grano $(\mathrm{G})(-10 /+20$ (grano I), -30 / +40 (grano II)); temperatura (T) $(800,900$ y $\left.1000^{\circ} \mathrm{C}\right)$ y tiempo (t) (10, 20 y 30 minutos).

Las variables dependientes son: rendimiento (\%), contenido de humedad ( $\mathrm{CH} \%)$, material volátil (MV \%), cenizas (\%), carbono fijo (CF $\%)$, capacidad de adsorción (Ads \%), valor de $\mathrm{pH}$ y densidad aparente $\left(\mathrm{g} / \mathrm{cm}^{3}\right)$.

Número de experimentos: $2^{\star}$ Tipo de granos ${ }^{\star} 3$ Temperaturas ${ }^{*} 3$ Tiempos $^{\star} 3$ Repeticiones $=54$ experimentos.

Con los resultados obtenidos se realizó el análisis de varianza (ANVA) para determinar el grado de influencia que ejercen las variables independientes sobre las dependientes y sus respectivas interacciones. En todos los casos se tomó como referencia el nivel de confianza con $95 \%$ de probabilidad $(\alpha=0,05)$ y de $99 \%$ $(\alpha=0,01)$ para determinar una influencia altamente significativa.

\section{Resultados}

\section{Caracterización del carbón vegetal}

En el Cuadro 1 se presentan los valores de los componentes químicos del carbón vegetal. Se obtuvo un rendimiento promedio de carbón vegetal de shapaja de 35,8\%. Respecto al contenido de humedad, se presenta un valor promedio de $4,7 \%$, considerado en el rango de carbones de calidad. El valor de contenido de cenizas resultó en 3,7\%. Asimismo, el material

\begin{tabular}{|c|c|c|c|c|c|c|}
\hline Carbonización & $\begin{array}{c}\text { Temperatura } \\
\left({ }^{\circ} \mathbf{C}\right)\end{array}$ & $\begin{array}{c}\text { Rendimiento } \\
(\mathbf{\%})\end{array}$ & $\mathbf{C H}(\%)$ & MV (\%) & CZ (\%) & CF (\%) \\
\hline $1^{\circ}$ & 500 & 32,7 & 6,12 & 19,4 & 2,9 & 77,8 \\
\hline $2^{\circ}$ & 500 & 38,8 & 3,28 & 20,3 & 4,4 & 75,4 \\
\hline \multicolumn{2}{|c|}{ Promedio } & 35,8 & 4,7 & 19,9 & 3,7 & 76,6 \\
\hline
\end{tabular}

Cuadro 1. Caracterización del carbón vegetal de shapaja (Attalea phalerata). 
volátil presentó un valor de 19,9\% y el contenido de carbono fijo de $76,6 \%$.

\section{Carbón activado}

El Cuadro 2 presenta los valores promedios del carbón activado de shapaja.

\section{Rendimiento}

Los rendimientos de activación del carbón granular y en polvo, disminuyen conforme aumenta la temperatura (Figura 1).

Con respecto al tiempo de activación, se observa que el rendimiento muestra una tendencia negativa al aumentar el tiempo para ambos tipos de carbón. Al respecto, el rendimiento disminuye porque una mayor fuente de calor, favorece la eliminación del material volátil.

\section{Contenido de humedad (CH)}

Los valores del contenido de humedad del carbón activado para el tipo granular y en polvo se presenta en la Figura 2. Al respecto el análisis de varianza presenta influencia altamente significativa en las tres variables de activación: grano $(\mathrm{G})$, tiempo $(\mathrm{T})$ y temperatura $(t)$; y sus interacciones $\left(G^{\star} T^{\star} t\right)$.

\section{Material volátil (MV)}

El contenido de material volátil presenta una tendencia negativa en función de la temperatura de activación para los dos tipos de carbón (Figura 3). En ambos casos el contenido de MV se reduce a medida que aumenta la temperatura de activación, debido a que el calor se transmite con mayor intensidad a las partículas de

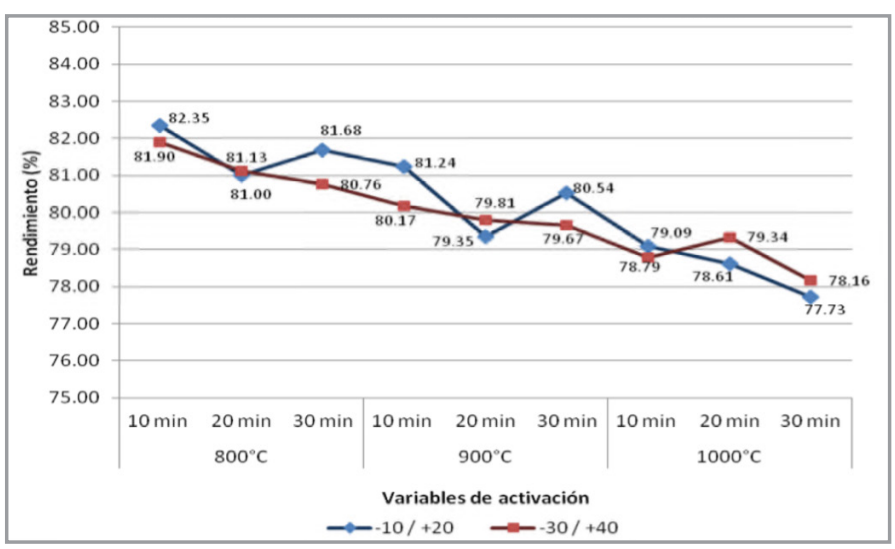

Figura 1. Rendimiento de activación para el grano I y II.

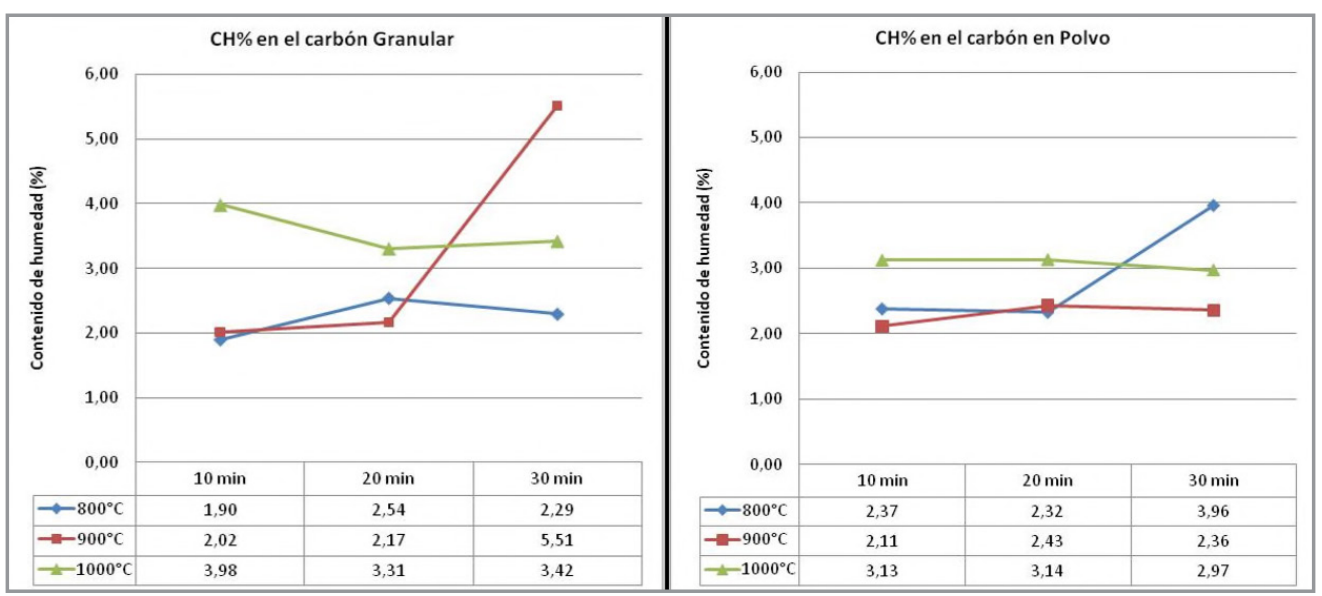

Figura 2. Variación del contenido de humedad $(\mathrm{CH})$ para el carbón granular y carbón en polvo. 


\begin{tabular}{|c|c|c|c|c|c|c|c|c|c|c|c|c|}
\hline $\begin{array}{l}\text { Tipo de } \\
\text { carbon }\end{array}$ & $\begin{array}{c}\text { Mues- } \\
\text { tra }\end{array}$ & Grano & $\underset{{ }^{\circ} \mathrm{C}}{\text { Temp. }}$ & $\begin{array}{c}\text { Tiem- } \\
\text { po } \\
\text { (min) }\end{array}$ & $\begin{array}{l}\text { Rendi- } \\
\text { mien- } \\
\text { to }(\%)\end{array}$ & $\begin{array}{l}\text { CH } \\
(\%)\end{array}$ & $\begin{array}{l}\text { MV } \\
\text { (\%) }\end{array}$ & $\begin{array}{l}\mathrm{CZ} \\
(\%)\end{array}$ & $\begin{array}{l}\text { CF } \\
(\%)\end{array}$ & $\begin{array}{c}\text { Ads } \\
\%\end{array}$ & $\begin{array}{c}\text { Dap } \\
(\mathrm{g} / \\
\left.\mathrm{cm}^{3}\right)\end{array}$ & pH \\
\hline \multirow{9}{*}{$\begin{array}{l}\text { Carbón } \\
\text { activado } \\
\text { granular } \\
\text { (grano I) }\end{array}$} & I-8-1 & \multirow{9}{*}{$-10 /+20$} & \multirow{3}{*}{800} & 10 & 82,3 & 1,9 & 7,3 & 3,1 & 89,6 & 60,5 & 0,61 & 6 \\
\hline & I-8-2 & & & 20 & 81,0 & 2,5 & 6,6 & 3,1 & 90,3 & 87,3 & 0,61 & 6 \\
\hline & I-8-3 & & & 30 & 81,7 & 2,3 & 6,5 & 3,0 & 90,4 & 83,9 & 0,61 & 6 \\
\hline & I-9-1 & & \multirow{3}{*}{900} & 10 & 81,2 & 2,0 & 5,9 & 3,2 & 90,9 & 95,6 & 0,62 & 6 \\
\hline & I-9-2 & & & 20 & 79,3 & 2,2 & 5,1 & 2,1 & 92,8 & 98,8 & 0,67 & 6 \\
\hline & I-9-3 & & & 30 & 80,5 & 5,5 & 6,2 & 2,8 & 90,9 & 96,6 & 0,60 & 6 \\
\hline & I-10-1 & & \multirow{3}{*}{1000} & 10 & 79,1 & 3,9 & 5,9 & 2,2 & 91,9 & 94,2 & 0,71 & 6 \\
\hline & I-10-2 & & & 20 & 78,6 & 3,3 & 4,7 & 2,5 & 92,7 & 97,1 & 0,70 & 6 \\
\hline & I-10-3 & & & 30 & 77,7 & 3,4 & 4,4 & 2,3 & 93,3 & 99,4 & 0,67 & 6 \\
\hline \multirow{9}{*}{$\begin{array}{c}\text { Carbón } \\
\text { activado } \\
\text { en polvo } \\
\text { (grano II) }\end{array}$} & II-8-1 & \multirow{9}{*}{$-30 /+40$} & \multirow{3}{*}{800} & 10 & 81,9 & 2,4 & 8,3 & 17,4 & 74,3 & 99,8 & 0,65 & 6 \\
\hline & II-8-2 & & & 20 & 81,1 & 2,3 & 7,8 & 14,3 & 77,9 & 99,6 & 0,69 & 7 \\
\hline & II-8-3 & & & 30 & 80,8 & 3,9 & 8,2 & 14,2 & 77,6 & 99,3 & 0,71 & 6 \\
\hline & II-9-1 & & \multirow{3}{*}{900} & 10 & 80,2 & 2,1 & 6,2 & 5,5 & 88,3 & 98,8 & 0,81 & 6 \\
\hline & II-9-2 & & & 20 & 79,8 & 2,4 & 6,4 & 5,2 & 88,2 & 99,3 & 0,81 & 6 \\
\hline & II-9-3 & & & 30 & 79,7 & 2,4 & 7,0 & 8,6 & 84,3 & 98,6 & 0,73 & 6 \\
\hline & II-10-1 & & \multirow{3}{*}{1000} & 10 & 78,8 & 3,1 & 4,9 & 13,5 & 81,7 & 99,5 & 0,75 & 6 \\
\hline & II-10-2 & & & 20 & 79,3 & 3,1 & 4,9 & 19,4 & 75,7 & 99,7 & 0,74 & 7 \\
\hline & II-10-3 & & & 30 & 78,2 & 2,9 & 5,5 & 19,6 & 74,9 & 99,6 & 0,74 & 7 \\
\hline
\end{tabular}

Cuadro 2. Valores promedios de las características del carbón activado obtenido a partir de endocarpio de shapaja (Attalea phalerata) por el método físico.

carbón, resultando en una mayor eliminación del material volátil.

Respecto al tiempo de activación (Figura 3), se observa que los valores de MV del carbón granular presenta una relación inversa, en tanto que el carbón en polvo una relación directa; sin embargo el coeficiente de determinación indica que sólo el $65,36 \%$ de las variaciones son explicadas por el tiempo.

\section{Contenido de cenizas $(\mathrm{CZ})$}

Los valores más altos de contenido de cenizas se encuentran en el carbón en polvo con 5,5 a $19,6 \%$ (Cuadro 2). Respecto a la temperatura de activación, se observa en la Figura 4 que influye significativamente en el carbón del tipo granular con tendencia negativa, reduciendo el valor del contenido de cenizas al aumentar la temperatura. Para el carbón en polvo no se estableció relación directa con la temperatura.
De acuerdo al tiempo de activación (Figura 4), no se encontró relación con el contenido de cenizas para el carbón granular; sin embargo existe una relación directa con el carbón en polvo. Esto se debe probablemente a diversas reacciones químicas, favorecidas por el tiempo de contacto entre el material carbonoso y el contenido de volátiles retenidos. El carbón granular presenta valores de cenizas menores a los especificados por la Norma Técnica Peruana NTP 207.024: 1982 y Mexicana NMX-F-295-1981. Asimismo, del análisis estadístico se desprende que todas las variables y sus interacciones presentan una influencia altamente significativa en el contenido de cenizas.

\section{Contenido de carbono fijo}

El contenido de carbono fijo presenta una influencia altamente significativa con las variables de estudio G, T y t, así como con sus 


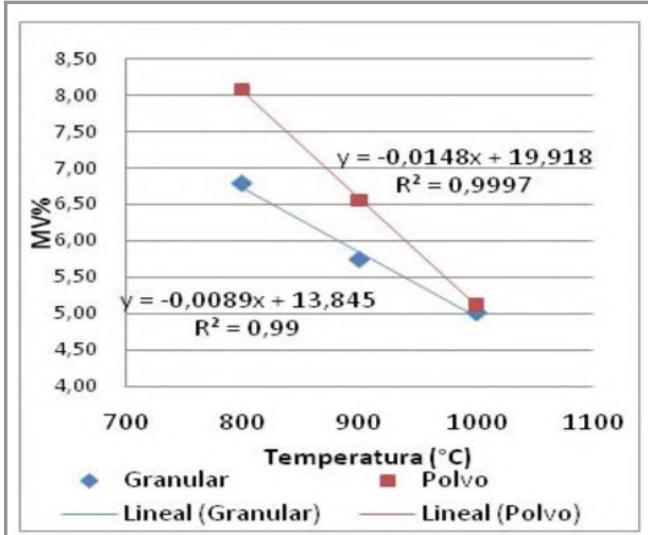

(A)

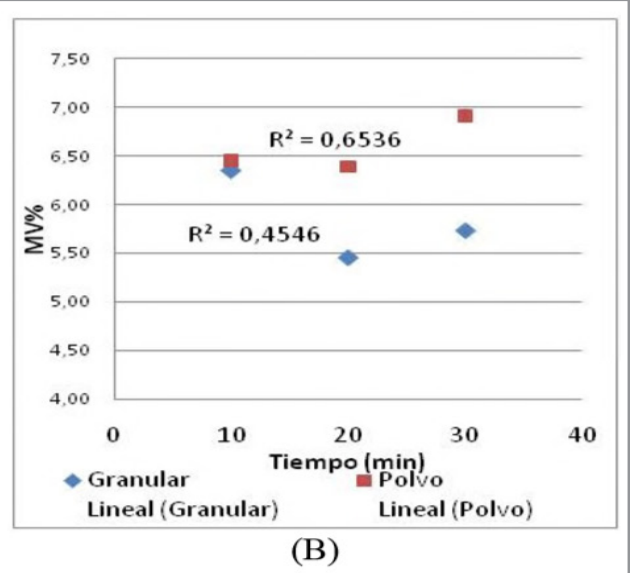

Figura 3. Variación del material volátil (MV) en función de la temperatura (A) y el tiempo de activación (B).

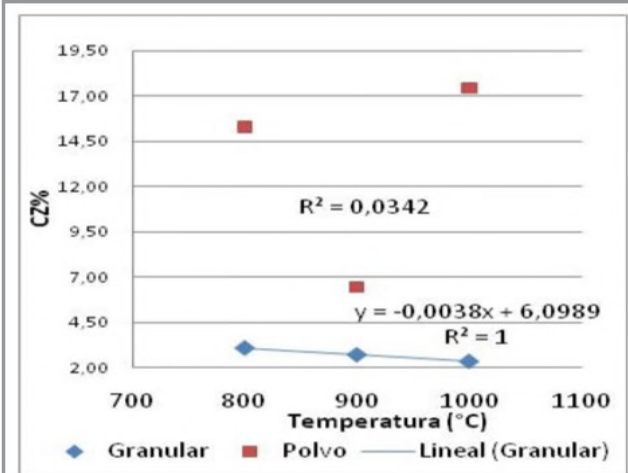

(A)

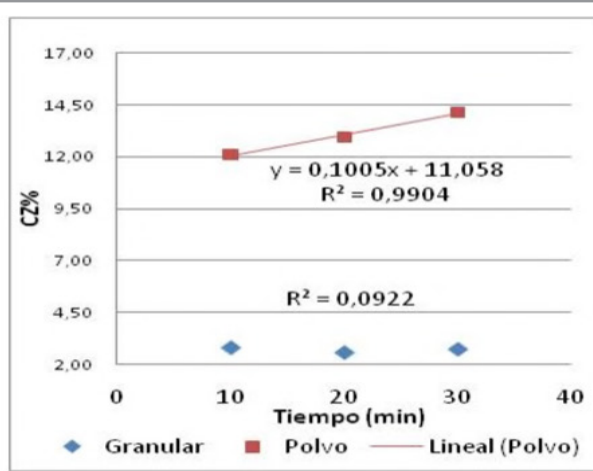

(B)

Figura 4. Variación del contenido de cenizas (CZ) en función de la temperatura (A) y el tiempo de activación (B).

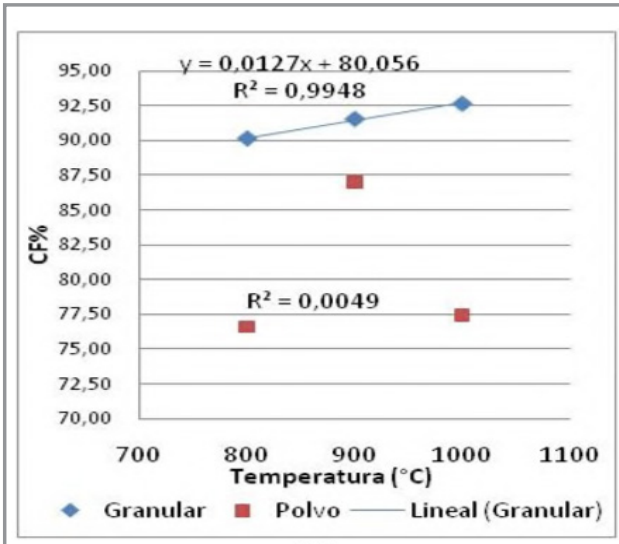

(A)

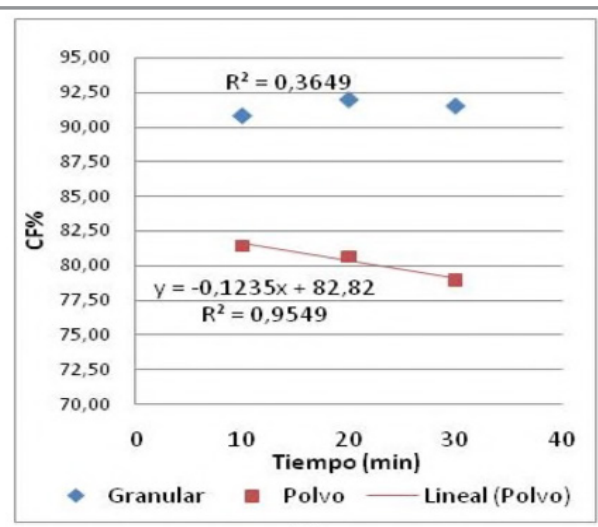

(B)

Figura 5. Variación del carbono fijo (CF) en función de la temperatura (A) y el tiempo de activación (B). 
interacciones $\left(G^{\star} T^{\star} t\right)$, cuyo coeficiente de determinación señala que el $99,3 \%$ de las variaciones del carbono fijo son explicadas por las variables $\mathrm{G}$, $\mathrm{T}$ y $\mathrm{t}$, del modelo experimental.

El carbón en polvo (grano II) presenta valores de 74,3 a 88,3\% menores comparado con el carbón granular (grano I) cuyos valores oscilan entre 89,6 a $93,2 \%$, señalándose que este último tendría buenas cualidades por su alto contenido de carbono fijo (Cuadro 2).

Por otro lado, se observa que la temperatura de activación presenta una relación directa con el CF en el carbón granular (Figura 5). Sin embargo, el carbón en polvo presenta una relación inversa con el CF, cuando se aumenta el tiempo de activación.

\section{Capacidad de adsorción}

En el análisis de ANVA se encuentra una influencia altamente significativa de las tres variables de estudio y sus interacciones. En el Cuadro 2, para el carbón granular se observa que a $800^{\circ} \mathrm{C}$ la capacidad de adsorción aumenta progresivamente con el tiempo; en cambio, el carbón a temperaturas mayores presentan un mayor nivel de adsorción, pero la influencia del tiempo es menor. En el carbón en polvo los valores de adsorción, en función del tiempo, son muy similares, no apreciándose diferencia importante. Asimismo se pudo observar que los valores de adsorción son mayores a menor tiempo de activación, incluso con mayor temperatura.

$\mathrm{Al}$ analizar la influencia de las variables temperatura y tiempo de activación, sobre la adsorción, para carbón granular (Figura 6), se observa que los resultados coinciden con lo expuesto por Román et al. (2008), Gonzales (2004) y Tongpoothorn et al. (2011). Quienes señalan que al elevar la temperatura y tiempo de activación se logra una mayor pérdida de material volátil, ampliando y generando una estructura más porosa y por ende de mayor área superficial y capacidad de adsorción. En el carbón en polvo, no se encontró relación con la temperatura, tiempo de activación y capacidad de adsorción.

\section{Densidad aparente}

El Cuadro 3 muestra los valores de densidad aparente $\left(\mathrm{D}_{\mathrm{ap}}\right)$ para el carbón granular y en polvo. Todos los valores son similares o cercanos entre sí, siendo los de carbón activado en polvo, ligeramente más altos, con un máximo de $0,81 \mathrm{~g} / \mathrm{cm}^{3}$.

En la Figura 7 se observa que la temperatura ejerce influencia directa sobre la densidad aparente del carbón granular. El tiempo, por el contrario, no presenta ninguna relación para los dos tipos de grano. El análisis estadístico demostró que las tres variables de activación influyen sobre la densidad aparente, con interacciones altamente significativas, con excepción de la interacción $\mathrm{G}^{*}$, que presentó una influencia no significativa.

\section{Valor de $\mathrm{pH}$}

El potencial de hidrógeno fue medido como un dato referencial y en la tabla de promedios (Cuadro 2) se puede apreciar que el $\mathrm{pH}$ del producto obtenido es neutro. $\mathrm{El} \mathrm{pH}$ de la mayoría de las unidades experimentales obtuvo un valor de 6 y solo tres de estas unidades, I-8-1, II-10-2 y II-10-3, marcaron un valor diferente, igualmente neutro, de 7 .

Según normas técnicas el valor de $\mathrm{pH}$ debe encontrarse entre 5,5 y 8 , rango que admite los resultados obtenidos por el carbón activado de shapaja del presente estudio, tanto del tipo granular como el tipo polvo. Estos valores se encuentran en el rango especificado por Norma Técnica Peruana NTP 207.024: 1982 y Mexicana NMX-F-295-1981 y productos comerciales como carbón activado Calgon. El valor de $\mathrm{pH}$ neutro favorece la producción pues no requeriría de tratamiento de lavado y secado para corregir esta variable.

\section{Selección del carbón activado}

Los resultados de los carbones activados obtenidos en el presente estudio, se compararon con las especificaciones exigidas para el uso de carbón activado granular en el tratamiento de agua de la NTP 311.331:1998: Carbón activado para tratamiento de agua para consumo humano y la Norma NMX-295-1981 para uso de 


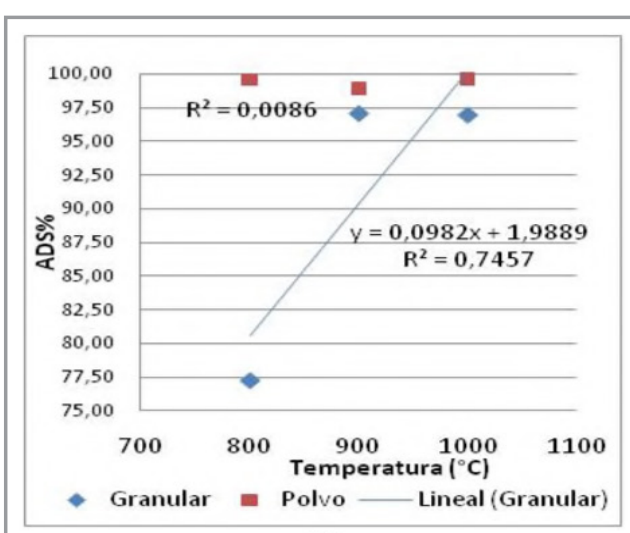

(A)

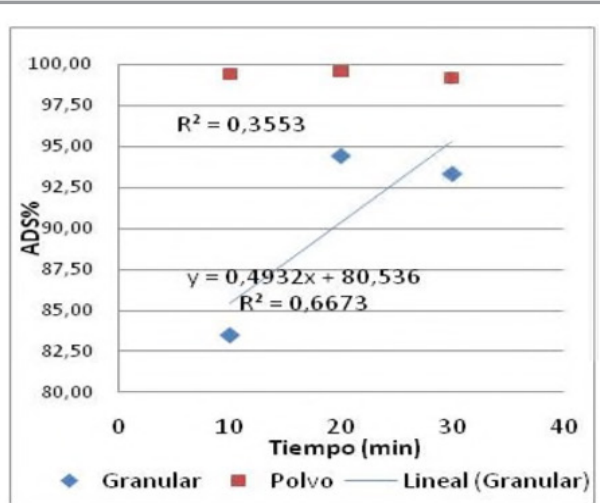

(B)

Figura 6. Variación de la capacidad de adsorción (ADS) (A) en función del la temperatura y tiempo.
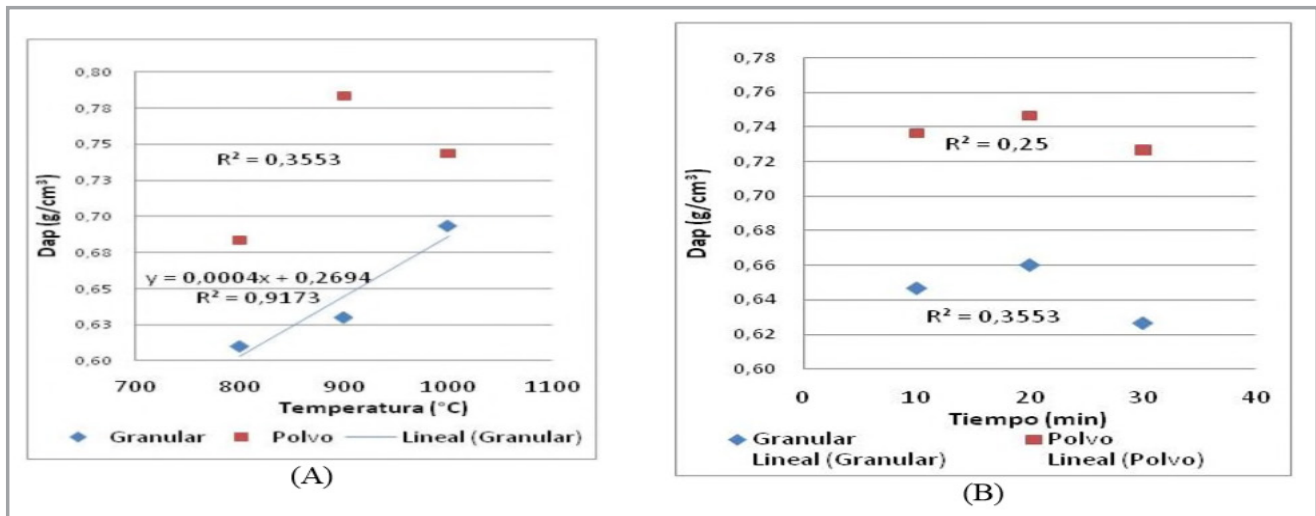

Figura 7. Variación de la densidad aparente (Dap) en función del la temperatura (A) y tiempo de activación (B).

carbón activado en la refinación de azúcar. Los resultados de estas evaluaciones se encuentran en el Cuadro 3.

Respecto a la a la Norma NMX-F-295-198, todos los parámetros evaluados de los carbones activados grano I y II se encuentran dentro del rango establecido para uso de carbón activado granular en tratamiento de agua, excepto los valores de densidad aparente, pues el resultado de menor magnitud es mayor al máximo valor permitido; para reducir el sesgo de este parámetro se valoraron los resultados de modo que los más cercanos a las referencias tuviesen un peso mayor en la elección.

En cuanto a la NTP 311.331:1998, los carbones activados grano I, cumplen con todos los parámetros evaluados excepto los valores de densidad aparente, por lo que se valoró el resultado más cercano al máximo exigido de $0,50 \mathrm{~g} / \mathrm{cm}^{3}$. Los carbones activados grano II, cumplen los parámetros de contenido de humedad y pH; y sólo 3 de ellos considerados en la elección, los valores de cenizas. Respecto a la densidad aparente, ninguno cumple con los valores que exige la norma, sin embargo se consideró el de menor densidad aparente.

De acuerdo con la evaluación efectuada, los carbones activados grano I $(-10 /+20$, Granular): I9-3 y grano II (-10/+20, Polvo): II-9-3, obtenido a $900^{\circ} \mathrm{C}$ y 30 minutos, tienen potencial para ser usados en el tratamiento de agua para consumo humano, así como en la refinación de azúcar. 


\begin{tabular}{|c|c|c|c|c|c|c|c|c|c|c|c|c|c|c|c|c|}
\hline Muestra & Grano & $\begin{array}{l}\text { CH } \\
(\%)\end{array}$ & $\begin{array}{l}\text { MV } \\
(\%)\end{array}$ & $\begin{array}{l}\text { CZ } \\
(\%)\end{array}$ & $\begin{array}{l}\text { CF } \\
(\%)\end{array}$ & Ads \% & $\begin{array}{c}\text { Dap } \\
(\mathrm{g} / \\
\left.\mathrm{cm}^{3}\right)\end{array}$ & $\mathrm{pH}$ & $\underset{<12}{\mathrm{CH} \%}$ & $\begin{array}{l}\mathrm{CZ} \% \\
<\mathbf{1 2 , 5}\end{array}$ & $\begin{array}{l}\text { Dap* } \\
<0,46\end{array}$ & $\begin{array}{c}\mathrm{pH} \\
5,5- \\
8\end{array}$ & $\begin{array}{l}\text { CH\% } \\
\text { Max12 }\end{array}$ & $\begin{array}{r}\text { CZ\% } \\
\text { Max12 }\end{array}$ & $\begin{array}{c}\text { Dap* } \\
\mathbf{0 , 3 5 -} \\
0,4\end{array}$ & $\begin{array}{c}\mathrm{pH} \\
\mathbf{5 , 5 -} \\
\mathbf{7 , 5}\end{array}$ \\
\hline I-8-1 & \multirow{9}{*}{$-10 /+20$} & 1,9 & 7,3 & 3,1 & 89,6 & 60,5 & 0,61 & 6 & + & + & + & + & + & + & + & + \\
\hline I-8-2 & & 2,5 & 6,6 & 3,1 & 90,3 & 87,3 & 0,61 & 6 & + & + & + & + & + & + & + & + \\
\hline I-8-3 & & 2,3 & 6,5 & 3,0 & 90,4 & 83,9 & 0,61 & 6 & + & + & + & + & + & + & + & + \\
\hline I-9-1 & & 2,0 & 5,9 & 3,2 & 90,9 & 95,6 & 0,62 & 6 & + & + & + & + & + & + & + & + \\
\hline I-9-2 & & 2,2 & 5,1 & 2,1 & 92,8 & 98,8 & 0,67 & 6 & + & + & + & + & + & + & + & + \\
\hline I-9-3 & & 5,5 & 6,2 & 2,8 & 90,9 & 96,6 & 0,60 & 6 & + & + & + & + & + & + & + & + \\
\hline I-10-1 & & 3,9 & 5,9 & 2,2 & 91,9 & 94,2 & 0,71 & 6 & + & + & + & + & + & + & + & + \\
\hline $\mathrm{I}-10-2$ & & 3,3 & 4,7 & 2,5 & 92,7 & 97,1 & 0,70 & 6 & + & + & + & + & + & + & + & + \\
\hline I-10-3 & & 3,4 & 4,4 & 2,3 & 93,3 & 99,4 & 0,67 & 6 & + & + & + & + & + & + & + & + \\
\hline II-8-1 & \multirow{9}{*}{$-30 /+40$} & 2,4 & 8,3 & 17,4 & 74,3 & 99,8 & 0,65 & 6 & + & - & - & + & + & - & - & + \\
\hline II-8-2 & & 2,3 & 7,8 & 14,3 & 77,9 & 99,6 & 0,69 & 7 & + & - & - & + & + & - & - & + \\
\hline II-8-3 & & 3,9 & 8,2 & 14,2 & 77,6 & 99,3 & 0,71 & 6 & + & - & - & + & + & - & - & + \\
\hline II-9-1 & & 2,1 & 6,2 & 5,5 & 88,3 & 98,8 & 0,81 & 6 & + & + & - & + & + & + & - & + \\
\hline II-9-2 & & 2,4 & 6,4 & 5,2 & 88,2 & 99,3 & 0,81 & 6 & + & + & - & + & + & + & - & + \\
\hline II-9-3 & & 2,4 & 7,0 & 8,6 & 84,3 & 98,6 & 0,73 & 6 & + & + & - & + & + & + & - & + \\
\hline II-10-1 & & 3,1 & 4,9 & 13,5 & 81,7 & 99,5 & 0,75 & 6 & + & - & - & + & + & - & - & + \\
\hline II-10-2 & & 3,1 & 4,9 & 19,4 & 75,7 & 99,7 & 0,74 & 7 & + & - & - & + & + & - & - & + \\
\hline II-10-3 & & 2,9 & 5,5 & 19,6 & 74,9 & 99,6 & 0,74 & 7 & + & - & - & + & + & - & - & + \\
\hline
\end{tabular}

Cuadro 3. Evaluación de los carbones activados obtenidos de acuerdo a las normas NMX-295-1981 yla norma NTP. Se calificó con un signo (+) si cumple con la especificaciones y con un signo (-) si el resultado se encuentra fuera del rango establecido.

\section{Discusión}

El rendimiento de carbón activado de shapaja resultó mayor a los obtenidos por Meléndez (2008), entre 25 y $33 \%$ y lo señalado por FAO (1983), quien sostiene que los rendimientos de carbonización a $500^{\circ} \mathrm{C}$, se encuentran alrededor de $30 \%$.

La FAO (1983), señala que el contenido de humedad debe encontrarse entre 5 y $15 \%$, de preferencia con valores menores al $10 \%$, y evitar pérdidas por desmenuzamiento. Asimismo, el contenido de cenizas, debe presentar valores entre 0,5 y $5 \%$, y uno de buena calidad, alrededor de 3\% de cenizas; por lo que el resultado de la presente investigación es aceptable.

El material volátil presentó un valor mayor al registrado por otros residuos similares como el carozo del durazno con un valor de 3\% (Checco 1985); pero en el rango de lo propuesto por la
FAO (1983), entre 5 y 40 \%; que señala además que un buen carbón vegetal puede presentar un valor aproximado de $30 \%$ en contenido de material volátil. De manera similar el contenido de carbono fijo de 76,6\%, resultó menor al carbón de carozo de durazno, de 92,5\%; pero dentro del rango mencionado por FAO (1983), entre 50 y $95 \%$.

Los rendimientos de carbón en polvo son menores que el granular; porque en partículas de menor tamaño, el calor se transmite con mayor rapidez, favoreciendo la eliminación del material volátil y disminución del rendimiento. Azañero (1988) señala que el rendimiento decrece con el incremento de la temperatura y el tiempo de activación.

A partir del rendimiento y siguiendo la fórmula de Dubini, se determinó que los carbones producidos son del tipo micro poroso, con 
grados de porosidad (GP) menores al 50\% y tamaño de poros menores a $2 \mathrm{~mm}$, favorables para ser usados en medios líquidos y en adsorción de gases (Vargas et al. 2010, Dubini citado por Azañero 1988, Lartey 1999).

En general, los contenidos de humedad reportados por estudios previos como los de Checco (1985) y Romero (1991) alcanzan contenidos de humedad de 2,2 y $2,72 \%$ respectivamente, ambos activados por medio físico, confirmando los valores obtenidos en el presente estudio. Por otro lado, Zamora (2010) en su investigación de activación química de shapaja registra valores menores, siendo el máximo contenido de humedad de 2,05\%. Respecto a los requisitos para usos industriales, los valores obtenidos son aceptables para la Norma Técnicas Mexicana, NMX-F-295-1981 y Norma Técnica Peruana NTP 207.024: 1982.

Los resultados de material volátil coinciden con lo descrito por Azañero (1988), Hassler (1974), Yang et al. (2010) y Tongpoothorn (2011). Además, son similares a los encontrados por Checco (1985) con 4,8\% y Romero (1991) con 9,02\%. En cambio los valores obtenidos por Zamora (2010), por el método químico, son mayores, entre 11 y $16 \%$.

Debido al proceso de activación, el contenido de MV disminuye de manera importante para los dos tipos de carbón; asimismo, el efecto combinado de tiempo y temperatura favorece la reducción del material volátil. Sin embargo, para el carbón activado en polvo, aumenta con el tiempo de activación, debido probablemente, a que el MV haya quedado retenido entre los poros de menor tamaño. Estadísticamente se demostró que todas las variables de activación, así como sus interacciones, presentan una influencia altamente significativa en los resultados del contenido de MV.

El contenido de cenizas coincide con lo descrito por Romero (1991) para carbón de cáscara de semilla de algodón y similares a los de Sangay (2010), para carbón activado de endocarpio de castaña con 12,5 \% y Zamora (2010) para carbón activado de aguaje con 7,19\%. Çuhadar (2005) menciona que a mayor conteni- do de CF se espera un área superficial mayor, y reporta que este contenido debe encontrarse entre 85 y $90 \%$ para asegurar la calidad del carbón.

Los valores obtenidos, para ambas granulometrías, son semejantes a los descritos por Checco (1985) y Romero (1991), con valores $90,8 \%$ y $85,3 \%$ respectivamente. En la activación química descrita por Sangay (2010), el CF sólo alcanzó 65,3\%; igualmente para Zamora (2010) los valores oscilan entre 65 y 80\%, menores a los obtenidos en el presente estudio.

La capacidad de adsorción coincide con los estudios de Arami-Niya et al. (2010) y Ould-Idriss et al. (2011), quienes mencionan que un aumento del tiempo conlleva a una reducción de la capacidad de adsorción pues los microporos se amplían formando meso y macroporos, los que a su vez reducen el área superficial antes formada.

El carbón activado de shapaja obtenido por medio químico presentó valores de adsorción entre 35 y 40 \% (Zamora 2010), menores a los presentado en este estudio. La activación física permitiría mayores valores de adsorción pues aumenta la formación de poros sin erosionar el carbón, como puede suceder con la activación química, logrando formación de una mayor área superficial.

La densidad aparente obtenida por activación química de shapaja (Zamora 2010), obtuvo valores promedio de $0,34 \mathrm{~g} / \mathrm{cm}^{3}$, menores a los obtenidos por la activación física; se explica porque en la activación química, la impregnación del reactivo, erosiona el carbón favoreciendo la pérdida de masa. Gómez et al. (2010) señalan que una pérdida importante de masa puede reducir los valores de densidad aparente, por lo que se puede inferir que la pérdida en la presente activación ha sido poco importante.

Los ensayos realizados por activación física de Checco (1985) y Romero (1985) presentan valores de $\mathrm{pH}$ superiores a 8 , siendo cercanos a los obtenidos en esta investigación. Se observa la diferencia con los activados por medio químico como los reportados por Layseca 
(2001) y Sangay (2010) de 3,19 y 4,55 respectivamente.

Las distintas propiedades del carbón activado son evaluadas a través de métodos analíticos normalizados por diversas asociaciones, aplicados para estandarizar y permitir al usuario comparar las especificaciones de diversos carbones (Ramírez 2009), sin embargo, se debe de tener en cuenta que no hay un solo tipo de carbón activado que sea el mejor para todas las aplicaciones (Schaeffer 2003).

El menor contenido de cenizas influyo en la selección de los carbones, por sus efectos en la capacidad de adsorción (Vargas et al. 2010), y es recomendable mantener bajos porcentajes del mismo (Çuhadar 2005). En capacidad de adsorción los referentes no se encuentran disponibles en las normas técnicas, sin embargo el resultado, se tomó en cuenta para optar por uno u otro carbón.

\section{Conclusiones}

El carbón de "shapaja" obtenido por destilación seca es un precursor con buenas características para la producción de carbón activado.

Los carbones obtenidos alcanzaron rendimientos de activación en el rango de 77,7 $-82,3$ y $78,2-81,9$ para la granulometría- $10 /+20$ $\mathrm{y}-30 /+40$ respectivamente.

Las pérdidas por combustión de los dos tipos de carbón son menores al 50\%, por lo que se espera que presenten una estructura mayormente microporosa.

Las variables granulometría, temperatura y tiempo, así como sus interacciones presentaron una influencia altamente significativa sobre todas sus características físicoquímicas, excepto con la interacción grano ${ }^{\star}$ tiempo, de la densidad aparente.

El contenido de cenizas y material volátil aumentan en el carbón activado en polvo, con el incremento del tiempo de activación, dando como resultado un menor contenido de carbono fijo.
La capacidad de adsorción con azul de metileno presenta una relación inversa con el tamaño de partícula: a mayor tamaño de partícula, menor porcentaje de adsorción.

Los carbones activados a partir de semillas de shapaja, con mayor potencial para ser utilizados en el tratamiento de agua y refinación de azúcar, fueron los carbones granular y en polvo, a $900^{\circ} \mathrm{C}$ y 30 minutos de activación.

\section{Bibliografía}

Arami-Niya, A; Daud, W; Mjalli, F. 2010. Using granular activated carbon prepared from oil palm shell by $\mathrm{ZnCl}_{2}$ and physical activation for methane adsorption. Journal of Analytical and Applied Pyrolysis 89(2): 197-203.

Azañero, I. 1988. Obtención de carbón a partir de la madera de algarrobo (Prosopis pallida HyB ex- Wild - HBK) mediante método físico. Tesis de Ingeniero en industrias alimentarias, Universidad Nacional Agraria La Molina, Lima, Perú.

Calgon Carbon Corporation. 2012. Product bulletin: GRC 20 and GRC 22, Gold Recovery Granular Activated Carbon. Consultado el 24 abr. 2012. Disponible en http://www.calgoncarbon.com/carbon_products/documents/ GRC_BUlletin_0621p.pdf

Castro, M. 2008. Babasú (Orbignya phalerata Mart.). Blog "Las materias primas para los biocarburentes". Consultado el 05 mar. 2011. Disponible en http://materiaprimas.blogspot.com/2008/07/ babau-orbignya-phalerata-mart.html

Checco, C. 1985. Obtención de carbón activo a partir del carozo de durazno (Prunus persica), Tesis de Ingeniero en industrias alimentarias, UNALM, Lima, Perú.

Çuhadar, Ç. 2005. Production and characterization of activated carbon from hazelnut shell and hazelnut husk. Tesis para obtener el grado de Magister. Graduate School of Natural and Applied Sciences, in partial fulfillment of the requirements for the Degree of Master of Science in Chemical Engineering, Middle East Technical University. 
FAO (Organización de las Naciones Unidas para la Alimentación). 1983. Métodos simples para fabricar carbón vegetal. Estudio FAO: Montes 41.

Fombuena, M. 2009. Manual del Carbón activo. Tesis para obtener el grado de Máster en Ingeniería del Agua. Universidad Politécnica de Sevilla, España.

Fredonia Group. 2010. World Activated Carbon. Industry Study with forecasts for 2014 \& 2019. Study\#2626, Junio. 379 p.

Gómez, A; Rincón, S; Klose, W. 2010. Carbón activado de cuesco de palma. Estudio de termo gravimetría y estructura. Facultad de Ingeniería de la Universidad Nacional de Colombia (Bogotá, Colombia), Facultad de Ingeniería Mecánica de la Universidad de Kasel (Kasel, Alemania).

Gonzales, I. 2004. Obtención de carbón activado a partir del endocarpio del fruto de la castaña (Bertholletia excelsa H.B.K) empleando el método químico con $\mathrm{ZnCl}_{2}$. Tesis de Ingeniero Forestal, Universidad Nacional Agraria La Molina, Perú.

Hassler, J. 1974. Purification with activated carbon: industrial commercial environmental, third ed., Chemical Publishing Co., New York.

INEI (Instituto Nacional de Estadística e Informática). 2011. Dirección técnica de demografía e indicadores sociales 2010. Perú: Anuario de Estadísticas Ambientales.

Lartey, R; Acquah, F; Nketia, K. 1999. Developing national capability for manufacture of activated carbon from agricultural wastes. The Ghana Engineer.

Layseca, M. 2001. Obtención de Carbón activado a partir de aserrío de Tornillo (Cedrelinga cateniformis Ducke), mediante el Método Químico ( $\mathrm{ZnCl} 2)$. Tesis de Ingeniero Forestal. Universidad Nacional Agraria La Molina, Perú.

Márquez, F; Carballo, C; Hernández, J. 2007. El carbón activado. Obtención y caracterización. Universidad de Pinar del Río, Cuba.

Menéndez, J. 2008. Curso de extensión universitaria: el mundo del residuo: caracterización, tratamiento, gestión. Tema: Residuos de biomasa para la producción de carbones activos y otros materiales de interés tecnológico. Universidad de Oviedo.

Norma NMX-F-295-1981. 1981. Alimentos para uso humano. Azúcar. Determinación de la calidad de carbones activados empleados en la refinación de azúcar. Foods for human use. Dirección general de normas, México.

Norma Técnica Peruana. 1982. NTP 207.024:1982, Carbón activado usado en la industria azucarera y otros productos edulcorantes - Requisitos generales.

Ould-Idriss, A; Stitou, M; Cuerda-Correa, E; Fernández-González, C; Macías-García, A; Alexandre-Franco, M; Gómez-Serrano, V. 2011. Preparation of activated carbons from olive-tree wood revisited. Physical activation with air. Fuel Processing Technology 92 (2): 266-270.

Performance Chemicals Inc. 2002. Carbón activado. Consultado el 04 may. 2012. Disponible en http://www.perfchem.com

Ramírez, C. 2009. El Carbón activado para el tratamiento del agua. Tesis Ing. Químico. Hermosillo, México, Universidad de Sonora.

Reynel, C; Pennington, R; Pennington, T; Flores, C; Daza, A. 2003. Árboles útiles de la Amazonía Peruana, un manual con apuntes de identificación, ecología y propagación de las especies. Tarea Gráfica Educativa.

Rodríguez, F. 2004. Carbón activado: Estructura, preparación y aplicaciones. Universidad de Alicante. España.

Román, S; González, J; González-García, C; Zamora, F. 2008. Control of pore development during $\mathrm{CO}_{2}$ and steam activation of olive stones. Fuel Processing Technology 89 (8): 715 -720 .

Romero, V. 1991. Obtención de carbón activado a partir de la cáscara de la semilla de algodón (Gossypium spp.) por activación química y su caracterización. Tesis de Ingeniero en Industrias Alimentarias. Universidad Nacional Agraria La Molina, Perú. 
Sangay, S. 2010. Obtención y evaluación de carbón activado del endocarpo de castaña (Bertholletia excelsa) procedente de plantación y de bosque natural, empleando un método químico. Tesis de Ingeniero Forestal. Universidad Nacional Agraria La Molina, Perú.

Schaeffer, K. 2003. Carbón activado: magia negra para tratamiento de agua. Agua Latinoamérica 3 (5). Consultado enero 2012. Disponible en: http://www.agualatinoamerica. com/docs/pdf/Intermedio.pdf

Trademap, 2010. Estadísticas de comercio para el desarrollo internacional de las empresas. Disponible en: http://www.trademap.org/stAbout_tradeMap.aspx

Tongpoothorn, W; Sriuttha, M; Homchan, P; Chanthai, S; Ruangviriyachai, C. 2011. Preparation of activated carbon derived from Jatropha curcas fruit shell by simple thermo-chemical activation and characterization of their physico-chemical properties. Chemical Engineering Research and Design 89(3): 335-340.
U.S. Army Corps of Engineers. 2001. Engineering and Design Adsorption Design Guide. Design Guide No. 1110-1-2 (March).

Vargas, J; Giraldo, L; Moreno-Piraján, J. 2010. Preparation of activated carbons from seeds of Mucuna mutisiana by physical activation with steam. Journal of Analytical and Applied Pyrolysis 89 (2): $307-312$.

Yang, K; Peng, J; Xia, H; Zhang, L; Srinivasakannan, C; Guo, S. 2010. Textural characteristics of activated carbon by single step $\mathrm{CO}_{2}$ activation from coconut shells. Journal of the Taiwan Institute of Chemical Engineers 41 (3): 367-372.

Zamora, G. 2010. Obtención de carbón activado a partir de semillas, de dos palmeras de la Amazonia peruana, "shapaja" (Attalea phalerata) y "aguaje" (Mauritia flexuosa). Tesis de Ingeniero Forestal. Universidad Nacional Agraria La Molina, Perú. 